\title{
HETEROGENEOUS INTEGRATION OF BIOMIMETIC ACOUSTIC MICROSYSTEMS
}

\author{
Andreas G. Andreou, David H. Goldberg \\ Eugenio Culurciello, Milutin Stanacevic \\ Gert Cauwenberghs
}

\author{
Electrical and Computer Engineering, \\ Johns Hopkins University, Baltimore, MD 21218 \\ andreou@jhu.edu
}

\section{Larry Riddle}

Signals Systems Corporation,
Severna Park, MD 21146

\begin{abstract}
We discuss biologically inspired devices and architectures for acoustic processing microsystems. We exploit state of the art integrated microsytems technologies to heterogeneously integrate electronics and micromechanical structures for acoustic sensing and signal processing.
\end{abstract}

\section{INTRODUCTION}

Natural sensors and sensory systems are marvels of microsystem integration. They are also remarkably efficient and effective in sensory communication and motor control tasks. In our quest to engineer "ears" and "eyes" for computers, we are taking hints from biology, and we are exploring alternative approaches to the ubiquitous symbolic digital processing paradigm. In doing so we have explored large scale analog computation [1], [2] exploiting the rich repertoire of computational functions available to us when we move away from the traditional view of a transistor as being just a simple switch. Our approach relies on the design of algorithms that match the natural computational primitives from the state-of-the-art microsystem technologies. Our work until now was limited VLSI technologies. In this paper we proposed architectures and structures that go beyond the mixed analogdigital VLSI paradigm and explore the domain of Mixed AnalogDigital-MEchanical (MAD-ME) VLSI processing.

\section{MEMS ACOUSTIC MICROSYSTEM}

Truly integrated sensory microsystems must incorporate the acoustic to electrical transducer elements on a chip. Advances in micromechanical system fabrication enable us to integrate not only arrays of acoustic pressure sensors, but acoustic pressure gradient sensors, accelerometers and even air particle flow sensors such as a hot-wire anemometer on a single die. Our ultimate goal is to integrate different physical structures that acquire information and shape the noise in such way to maximize the capacity of a physical channel to specific sources of information such as clicks, tones or more complex acoustic patterns. Note that our aim is not the

This work was supported by DARPA/ONR MURI N00014-95-1-409 and a DARPA/ONR contract N00014-00-C-0315 Intelligent and NoiseRobust Interfaces for MEMS Acoustic Sensors precise restitution of signals by building just a better and more integrated microphone but rather to maximize the amount of reliable information for identification,classification and recognition tasks. A synthesis framework to perform this task in sensory microsystems can be found in [3].

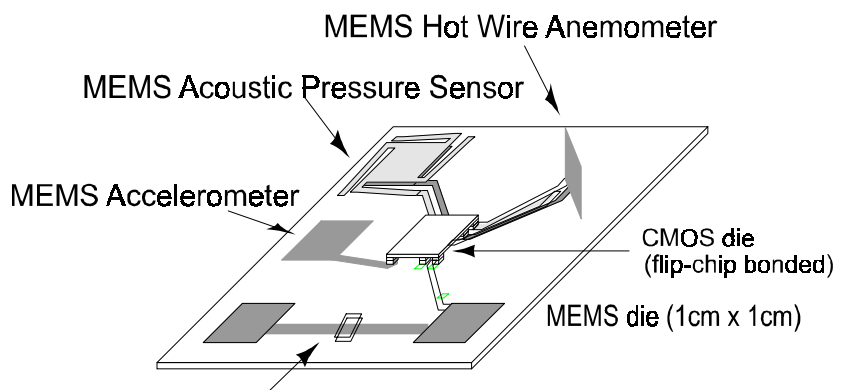

MEMS Acoustic Pressure Gradient Sensor

Figure 1: Proposed integrated acoustic microsystem. A $2 \times 2 \mathrm{~mm}^{2}$ CMOS chip is flip-chip bonded onto a $1 \times 1 \mathrm{~cm}^{2}$ MEMS die.

\subsection{Acoustic pressure sensor}

The simplest acoustic pressure sensor that we have designed is based on a capacitive detection principle. It consists of a polysilicon diaphragm suspended over a polysilicon backplate (Figure ??). This gives a capacitor with air as the dielectric. The suspended diaphragm is anchored to the substrate (nitride on silicon) via four serpentine spring supports. The dimensions of the supports determine the frequency response and sensitivity of the sensor rather than the shape or size of the diaphragm.

Because the springs are fixed at one end and free at the other, they can be modeled as cantilevers. The springs are all in parallel, so total stiffness is four times the stiffness of one spring:

$$
k=4 \frac{E W t^{3}}{4 L^{3}}
$$

where $E$ is the Young's modulus, $W$ is the cantilever width, $t$ is the thickness, and $L$ is the length. 


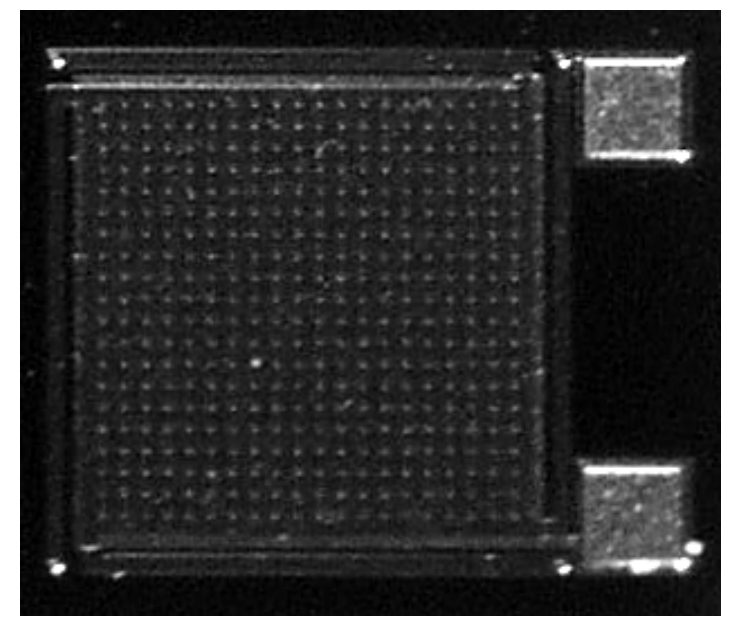

Figure 2: Photograph of the acoustic pressure sensor fabricated in the Cronos MUMPs ${ }^{\mathrm{TM}}$ process.

The resonant frequency is given by

$$
\omega_{0}=\sqrt{\frac{k}{m}}
$$

where $m$ is the mass of the diaphragm.

The pull-in voltage of the diaphragm is given by

$$
V_{P I}=\sqrt{\frac{8 k x_{0}^{3}}{27 \epsilon_{0} A}}
$$

where $x_{0}$ is the capacitive plate distance at zero volts and zero spring extension and $A$ is the area of the diaphragm.

The mechanical sensitivity of the sensor is defined as the increase in the deflection of the diaphragm, $d w$ where $x$ is the plate distance, resulting from an increase in pressure, $d P$ :

$$
S_{m}=\frac{d w}{d P}
$$

If we model the diaphragm as a simple spring with the equation $F=k w$, then

$$
\begin{aligned}
A d P & =k d w \\
S_{m}=\frac{d w}{d P} & =\frac{A}{k}
\end{aligned}
$$

$S_{m}$ has units of $\mathrm{m} / \mathrm{Pa}$. From Equation 6 we see that a sensitive sensor has a large area and a flexible diaphragm.

Two acoustic sensors like the one shown in Figure ?? were fabricated in the Cronos MUMPs ${ }^{\mathrm{TM}}$ process. The design parameters and estimated characteristics are given in Table 1 . Mic 1 had a Poly 1 diaphragm and Mic 2 had a Poly 2 diaphragm. The extremely low pull-in voltages are a consequence of low $k$ values, which may arise from the crude cantilever model of the springs. We are currently developing a more accurate model.

Reading out the small signal from the change in capacitance is achieved using a high impedance self biased floating gate MOS amplifier [4]. The high impedance sensing method also alleviates diaphragm collapse problems.

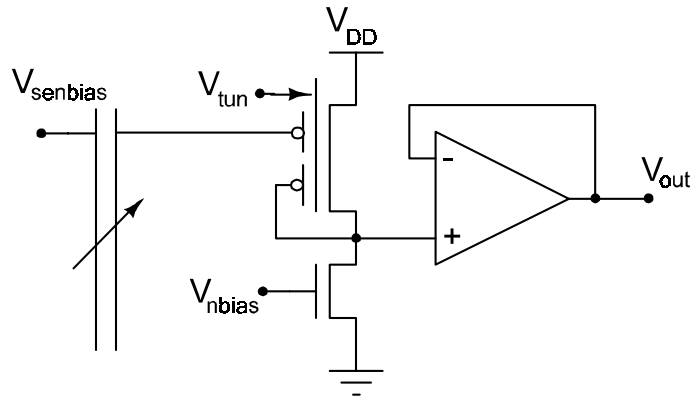

Figure 3: Readout amplifer using a self biased floating gate MOS amplifier

\begin{tabular}{|c|c|c|c|}
\hline Parameter & Mic 1 & Mic 2 & Units \\
Membrane area $(A)$ & $500 \times 500$ & $500 \times 500$ & $\mu \mathrm{m}^{2}$ \\
Membrane thickness $(t)$ & 2 & 1.5 & $\mu \mathrm{m}$ \\
Membrane mass $(m)$ & 1.17 & 0.874 & $\mu \mathrm{g}$ \\
Stiffness $(k)$ & 0.182 & 0.0768 & $\mathrm{~N} / \mathrm{m}$ \\
Resonant frequency $\left(\omega_{0}\right)$ & 12.5 & 9.37 & $\mathrm{kHz}$ \\
Air gap $\left(s_{0}\right)$ & 2 & 2.75 & $\mu \mathrm{m}$ \\
Pull-in voltage $\left(V_{P I}\right)$ & 0.442 & 0.463 & $V$ \\
Sensitivity $\left(S_{m}\right)$ & $1.37 \times 10^{-6}$ & $3.25 \times 10^{-6}$ & $\mathrm{~V} / \mathrm{Pa}$ \\
\hline
\end{tabular}

Table 1: Comparison of two different MEMS acoustic pressure sensors fabricated in the Cronos MUMPs ${ }^{\mathrm{TM}}$ process

\subsection{Mechanically-coupled acoustic pressure gradient sensor}

A more ambitious design is a biologically inspired acoustic pressure gradient sensor that is to be used in an array of such gradient sensors in conjuction with the independent component analysis system for broad band signal localization [5]. The microsensor is based on the mechanically-coupled acoustic sensory organs of the parasitoid fly Ormia ochracea [6]. Pardo and co-workers at Lucent Technologies [7] were the first to implement such a structure in the MUMPs ${ }^{\text {TM }}$ process.

The fly has remarkable directional hearing despite the fact that its two acoustic sensory organs are only $\sim 1 \mathrm{~mm}$ apart, corresponding to a 1 to $2 \mu \mathrm{s}$ difference in the sound pressure arrival times. Because the organs are coupled and they collectively pivot about the center (Figure 4), the system is very sensitive to the sound pressure differences between the two acoustic organs. Miles et al. conducted a detailed analysis of this system to show that the acoustic organs achieve directional hearing by amplifying interaural and level and time differences (ILD and ITD) through mechanical coupling [6].

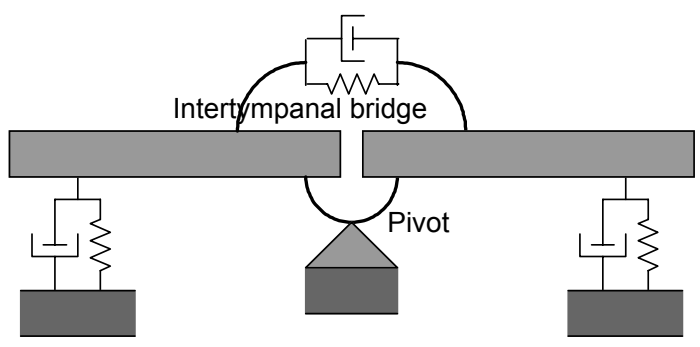

Figure 4: 
Our MEMS version consists of two acoustic sensors, each similar to the first design, connected by a Poly1 beam (Figure 5). The beam pivots about the substrate by means of an axle that is stapled to the substrate by two strips of Poly2 that are anchored to the substrate.

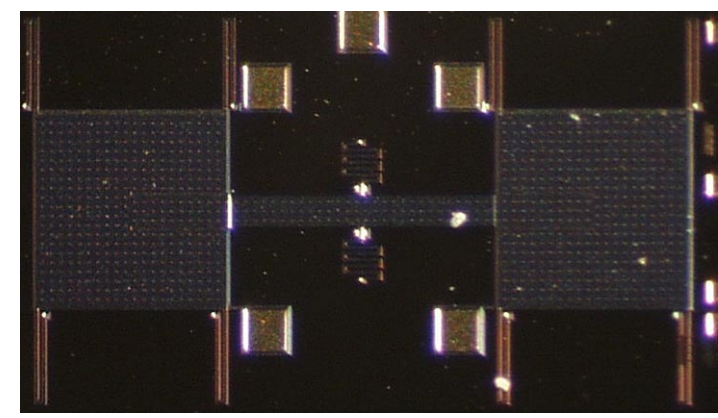

Figure 5: Acoustic pressure-gradient sensor

Designers of conventional MEMS microphones use bulk micromachining to create the backplate out of the substrate, complete with with acoustic holes. Then, they use surface micromachining to add the diaphragm. This permits a low resistance path for the air to travel from the gap between the plates into a large backchamber. In the case of our sensors, the limited path for air to escape from the gap between the plates will result in squeezed-film damping. One way to circumvent this problem in an all-surface micromachined design to create a backchamber by raising a tent-like structure out of the plane of the substrate [8]. Design of this kind of sensor structures is a focus of current investigation.

\section{MICROMECHANICAL SILICON COCHLEA}

The cochlea is the organ of hearing for humans. Sound waves that reach the eardrum are mechanically transferred to the cochlea which is a fluid-filled chamber partitioned by the basilar membrane. The mechanical vibrations create standing waves in the cochlear chamber that cause the basilar membrane to vibrate at frequencies corresponding to the incident acoustic wave frequency. For each frequency value there exists a location along the basilar membrane where the vibration is strongest. These locations roughly follow logarithmic ordering in frequency along the basilar membrane. Hence, in a generic form, the basilar membrane can be modeled as a bank of frequency selective filters (where the center-frequency of each filter is equally spaced on a log-scale), each representing a particular location that are equally spaced along the membrane $[9,10]$.

Preprocessing the acoustic signals to distribute the information in parallel channels is done using a cochlea filterbank $[1,10,11]$. A micro-electromechanical filter bank was designed and is in fabrication in the MUMPs ${ }^{\text {TM }}$ process provided by Cronos Integrated Microsystems. The MEMS silicon cochlea is composed by a bank composed by 11 filters logarithmically spaced in a bandwidth of $10 \mathrm{KHz}$. The log-spaced filter bank allows to derive the wavelet transform of the input acoustic signal in real time. Individual filters are obtained using laterally driven polysilicon resonant microstructures. Interdigitated comb structures can be electrostatically driven by input signal, and drive a polysilicon mass to resonate parallel to the substrate [12]. The frequency of resonance of each structure depends on the length of the mass-suspending cantilevers. A bias signal for the resonant structure can finely tune their quality factor, thus allowing modulation of the filtered bandwidth [13]. The advantage of a MEMS cohclear compared to an analog VLSI cochlea is lower power dissipation as the energy that normally goes in linearizing the transconductors and setting the parameters of the electronic cochleas is now conserved.

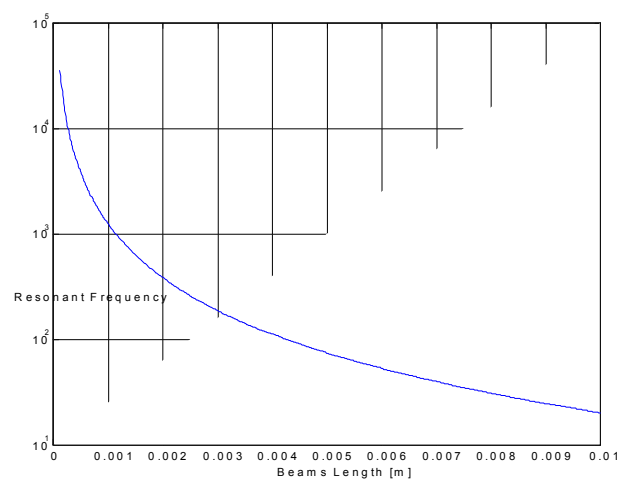

Figure 6: Filter bins (left) and the logarithmically spaced cutoff frequencies (right).

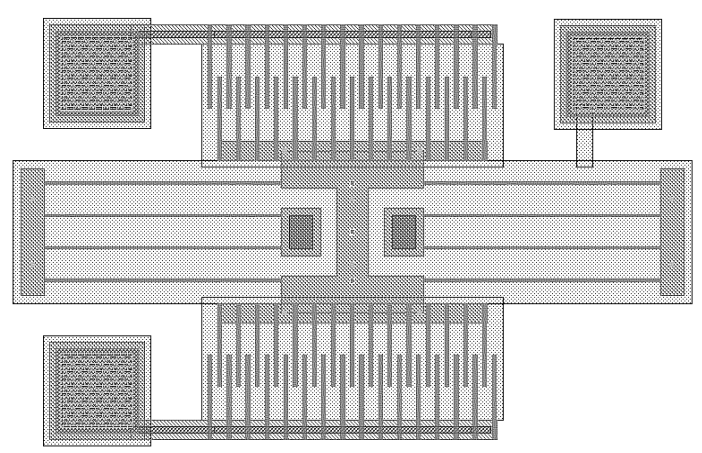

Figure 7: Interdigitated comb structure structures.

\section{FEATURE EXTRACTION}

The output of the silicon MEMS cochlea can be processed to convert the analog displacement into a discrete value continuous time signal representation [14] or can drive an independent component analysis module. The independent component analysis or HJ network is a neuromorphic architecture that has been employed for signal separation. For task of blind separation of linear convolutive mixtures, the solutions have been proposed in both time [15], and frequency domain $[16,17]$. The solutions are based on the inversing the mixing matrix of FIR filters. The length of unmixing filter has to be greater than the length of the mixing filter, leading to large number of taps (e.g. room impulse response for speech signal separation). The solution to decreasing the number of taps would be by introducing the all-pass filters instead of delay elements, which will match the characteristics of room impulse response more closely, 


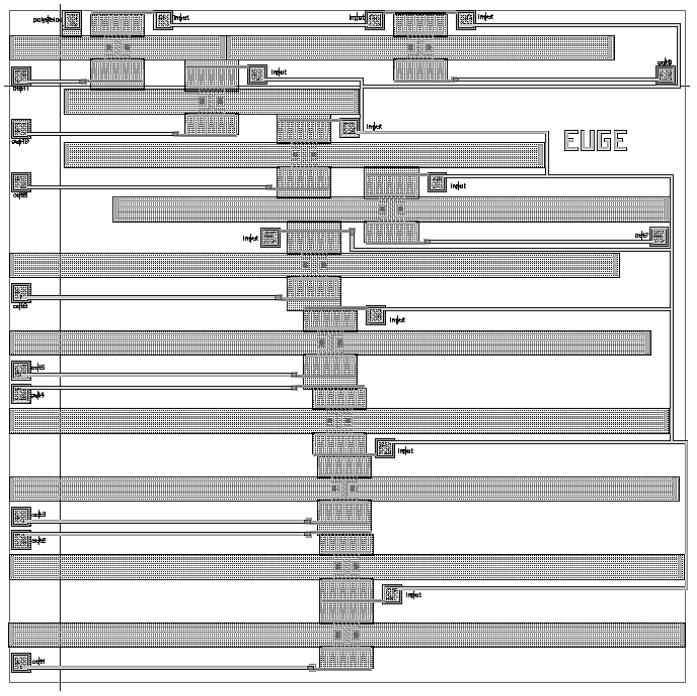

Figure 8: Layout of the MEMS filter bank, on an area of $2.4 \times 2.4 \mathrm{~mm}$.

and as result, we would get less number of elements needed for inverting the FIR filter [18].

\section{CONCLUSIONS}

In this paper we have presented electromechanical based computational structures and algorithms for acoustic processing. These microsystems will include biasing circuits, as well as preamplifiers and preprocessing signal processing as well as micromechanical structures such as those depicted in Figure 1. A $2 \times 2 \mathrm{~mm}^{2}$ CMOS chip is flip-chip bonded onto a $1 \times 1 \mathrm{~cm}^{2}$ MEMS die incorporates an acoustic pressure sensor, acoustic gradient sensors, an accelerometer and a hot-wire anemometer to measure the ambient wind conditions.

The heterogenous integration approach is a good compromise between the benefits of full integration and modular processing. As technological advances enable the manufacturing of integrated systems that incorporate basic elements other than electronic switches and wires, we will see a proliferation of complex computational devices that are structurally diverse and are heterogeneously integrated to achieve new levels of functionality and energy efficiency.

\section{REFERENCES}

[1] Carver Mead, 'Analog VLSI and Neural Systems', AddisonWesley Publishing, 1980.

[2] A.G. Andreou, "On physical models of neural computation and their analog VLSI implementation," Proceedings of the 1994 Workshop on Physics and Computation, IEEE Computer Society Press, pp 255-264, Los Alamitos, CA, 1994.

[3] A.G. Andreou and P. Abshire, "Sensory microsystems as communication networks: analysis and synthesis framework," Electrical and Computer Engineering Technical Report JHUECE 01-01, Johns Hopkins University, January 2001.
[4] P. Hasler, B. A. Minch, C. Diorio, and C. Mead, An autozeroing amplifier using pFET hot-electron injection, in IEEE International Symposium on Circuits and Systems, vol. 3, (New York), pp. 325328, IEEE, 1996.

[5] G. Cauwenberghs, M. Stanacevic and G. Zweig, "Blind Broadband Separation and Localization in Miniature Sensor Arrays," IEEE International Symposium on Circuits and Systems, May 5-8, 2001.

[6] R. Miles, D. Robert, and R. Hoy, Mechanically coupled ears for directional hearing in the parasitoid y ormia ochracea," Journal of the Acoustical Society of America, vol. 98, no. 6, pp. 3059-3070, 1995.

[7] F. Pardo, D. J. Bishop, P. Gammel, D. Lopez, B. Boie, G. Elko, and R. Sarpeshkar, All surface micromachined microphone." Available at http:www.darpa.milmto/sono- presentationslucentpardo.pdf.

[8] F. Pardo, R.Boie, G.Elko, R.Sarpeshkar, and D.Bishop, Allsurface-micromachined Si microphone," in TRANSDUCERS '99, 1999.

[9] Xiaowi Yang, Kuansan Wang, and Shihab Shamma, "Auditory representations of acoustic signals", IEEE Transactions on Information Theory, vol. 38, no. 2, pp. 824-839, March 1992.

[10] W. Liu, A. G. Andreou, and M. G. Goldstein, "Voiced speech representation by an analog silicon model of the auditory periphery", IEEE Transactions on Neural Networks, vol. 3, no. 3, pp. 477-487, May 1992.

[11] P. M. Furth and A. G. Andreou, "A design framework for low power analog filter banks," in IEEE Transactions on Circuits and Systems, Part I: Fundamental Theory and Applications, Vol. 42, No. 11, pp. 966-971, November 1995.

[12] W. C. Tang, T. H. Nguyen, R. T. Howe, 'Laterally Driven Polysilicon Resonant Microstructures', Sens. Actuators, Vol. 20, pp. 25-32, 1989.

[13] C. T. C. Nguyen, ", IEEE Trans. On Microwave Theory and Techniques, Vol. 47, No. 8, Aug. 1999.

[14] Nagendra Kumar, Gert Cauwenberghs, and Andreas G. Andreou, "A circuit model of hair-cell transduction for asynchronous analog auditory feature extraction", in International Symposium on Circuits and Systems. IEEE, 1996, vol. 3, pp. 301-304.

[15] M. Cohen and G. Cauwenberghs, "Blind Separation of Linear Convolutive Mixtures through Parallel Stochastic Optimization," IEEE International Symposium on Circuits and Systems, vol. 3, pp 17-20, 1998.

[16] R. Lambert and A. Bell, "Blind Separation of Multiple Speakers in a Multipath Environment," Proceedings of ICASSP, Munich, 1997.

[17] T.W. Lee, T. Bell and R. Orglmeister, "Blind Source Separation of Real World Signals," IEEE International Conference on Neural Networks, Houston, 1997.

[18] J.G. Harris, J.K. Juan and H.C. Principe, "Analog Hardware Implementation of Adaptive Filter Structures," Proc. of International Conference on Neural Networks, Houston, 1997. 